\title{
FLU, an amino acid substitution model for influenza proteins
}

\author{
Cuong Cao Dang ${ }^{1}$, Quang Si Le*2, Olivier Gascuel ${ }^{3}$ and Vinh Sy Le
}

\begin{abstract}
Background: The amino acid substitution model is the core component of many protein analysis systems such as sequence similarity search, sequence alignment, and phylogenetic inference. Although several general amino acid substitution models have been estimated from large and diverse protein databases, they remain inappropriate for analyzing specific species, e.g., viruses. Emerging epidemics of influenza viruses raise the need for comprehensive studies of these dangerous viruses. We propose an influenza-specific amino acid substitution model to enhance the understanding of the evolution of influenza viruses.
\end{abstract}

Results: A maximum likelihood approach was applied to estimate an amino acid substitution model (FLU) from 113, 000 influenza protein sequences, consisting of $\sim 20$ million residues. FLU outperforms 14 widely used models in constructing maximum likelihood phylogenetic trees for the majority of influenza protein alignments. On average, FLU gains $\sim 42 \mathrm{log}$ likelihood points with an alignment of 300 sites. Moreover, topologies of trees constructed using FLU and other models are frequently different. FLU does indeed have an impact on likelihood improvement as well as tree topologies. It was implemented in PhyML and can be downloaded from ftp://ftp.sanger.ac.uk/pub/1000genomes/lsq/ $\underline{F L U}$ or included in PhyML 3.0 server at http://www.atgc-montpellier.fr/phyml/.

Conclusions: FLU should be useful for any influenza protein analysis system which requires an accurate description of amino acid substitutions.

\section{Background}

The majority of statistical methods used for analyzing protein sequences require an amino acid substitution model to describe the evolutionary process of protein sequences. Amino acid substitution models are frequently used to infer protein phylogenetic trees under maximum likelihood or Bayesian frameworks [[1,2], and references therein]. They are also used to estimate pairwise distances between protein sequences that subsequently serve as inputs for distance-based phylogenetic analyses [3]. Moreover, these models can be used for aligning protein sequences [4]. These and other applications of the amino acid substitution model are reviewed in [5].

Many methods have been proposed to estimate general amino acid substitution models from large and diverse databases [[1,6], and references therein]. These methods

* Correspondence: Isq@sanger.ac.uk

2 Wellcome Trust Sanger Institute, Wellcome Trust Genome Campus, Hinxton, Cambridge, CB10 1SA, UK

Full list of author information is available at the end of the article belong to either counting or maximum likelihood approaches. The first counting method was proposed by Dayhoff et al. [7] to estimate the PAM model. As more protein sequences accumulated, Jones et al. [8] used the same counting method to estimate the JTT model from a larger protein data set. However, the counting methods are limited to only closely related protein sequences.

The maximum likelihood method was proposed by Adachi and Hasegawa [9] to estimate the mtREV model from 20 complete vertebrate mtDNA-encoded protein sequences. The mtREV model outperformed other models when analyzing the phylogenetic relationships among species based on their mtDNA-encoded protein sequences. Whelan and Goldman [10] proposed a maximum likelihood method to estimate the WAG model from 182 globular protein families. The WAG model produced better likelihood trees than the Dayhoff and JTT models for a large number of globular protein families.

Recently, Le and Gascuel [6] improved the maximum likelihood method by incorporating the variability of evolutionary rates across sites into the estimation process. 
The method was used to estimate the so-called LG model from the Pfam database. Experiments showed that the LG model gave better results than other models both in terms of likelihood values and tree topologies.

Although a number of general models have been estimated from large and diverse databases comprising multiple genes and a wide range of species, they might be inappropriate for a particular set of species due to differences in the evolutionary processes of these species. A number of specific amino acid substitution models for important species have been introduced [11,12], e.g. HIV-specific models that showed a consistently superior fit compared with the best general models when analyzing HIV proteins.

In recent years, the world has encountered a series of emerging influenza epidemics, including H5N1 ('avian flu') or H1N1. These have caused serious problems in economics and human health. Theoretical and experimental studies have been extensively conducted to understand the evolution, transmission and infection processes of influenza viruses [13-17]. We propose here our FLU model which was specifically estimated for modeling the evolution of influenza viruses. Experiment results show that FLU is robust and better than other models in analyzing influenza proteins. Thus, it could enhance studies of the evolution of influenza viruses.

\section{Results and Discussion}

We used the maximum likelihood approach introduced by Le and Gascuel [6] to estimate an influenza-specific amino acid substitution model (called FLU) from data set D comprising 992 influenza protein alignments. In the following sections, the main properties and performance of FLU in comparison with 14 widely used models will be analyzed.

\section{Model analysis}

FLU, as an amino acid substitution model, includes a symmetric amino acid exchangeability matrix and an amino acid frequency vector. Thus, we analyze FLU with other models by comparing their amino acid exchangeabilities and frequencies. Table 1 presents low correlations between FLU and other models, which means that FLU is highly different from existing models. HIVb and $\mathrm{HIVw}$ are among the models that are most highly correlated with FLU, since they were also estimated from RNA virus proteins.

In the following, we compare FLU with HIVb (a HIVspecific model) and LG (the best general model) in detail. Figure 1 displays the amino acid frequencies of these models and the empirical amino acid frequencies (denoted Influenza) that were counted from all alignments of data set $\mathbf{D}$. Amino acid frequencies of FLU and Influenza are nearly identical (correlation $~ 0.94$ ), the cor-
Table 1: The Pearson's correlations between FLU and 14 widely used models. The low correlations indicate that FLU is highly different from existing models.

\begin{tabular}{lll}
\hline model & $\begin{array}{c}\text { exchangeability } \\
\text { matrix }\end{array}$ & $\begin{array}{c}\text { frequency } \\
\text { vector }\end{array}$ \\
\hline $\mathrm{JTT}$ & 0.88 & 0.79 \\
$\mathrm{HIVb}$ & 0.86 & 0.71 \\
$\mathrm{HIVw}$ & 0.83 & 0.83 \\
WAG & 0.83 & 0.76 \\
LG & 0.82 & 0.71 \\
CpREV & 0.81 & 0.73 \\
Blosum62 & 0.77 & 0.73 \\
MtREV & 0.77 & 0.48 \\
RtREV & 0.76 & 0.66 \\
VT & 0.75 & 0.76 \\
MtMam & 0.74 & 0.48 \\
DCMut & 0.74 & 0.69 \\
Dayhoff & 0.74 & 0.69 \\
MtArt & 0.70 & 0.45 \\
\hline
\end{tabular}

relation being much higher than that of Influenza with the 2 other models, HIVb ( 0.84) and LG ( 0.84). Notably, we observe large differences between the amino acid frequencies of Influenza and the others. For example, the frequency of leucine $(\mathrm{L})$ in Influenza $(\sim 7 \%)$ is much lower than that in HIVb $(\sim 10 \%)$ and LG $(\sim 10 \%)$. These results indicate that FLU represents the amino acid frequencies of influenza proteins more accurately than other models.

The exchangeability coefficients of FLU, HIVb, and LG models (Figure 2), in principle, describe similar biological, chemical and physical properties of the amino acids, e.g. the high exchange rate between lysine (a positively charged, polar amino acid) and arginine (a positively charged, polar amino acid) or the low exchange rate

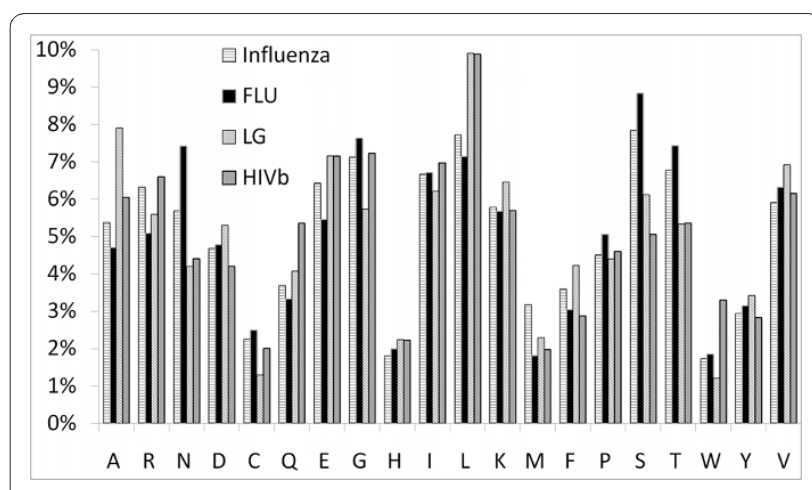

Figure 1 Amino acid frequencies of FLU, HIVb, LG models and the empirical frequencies counted from all alignments (denoted Influenza). 


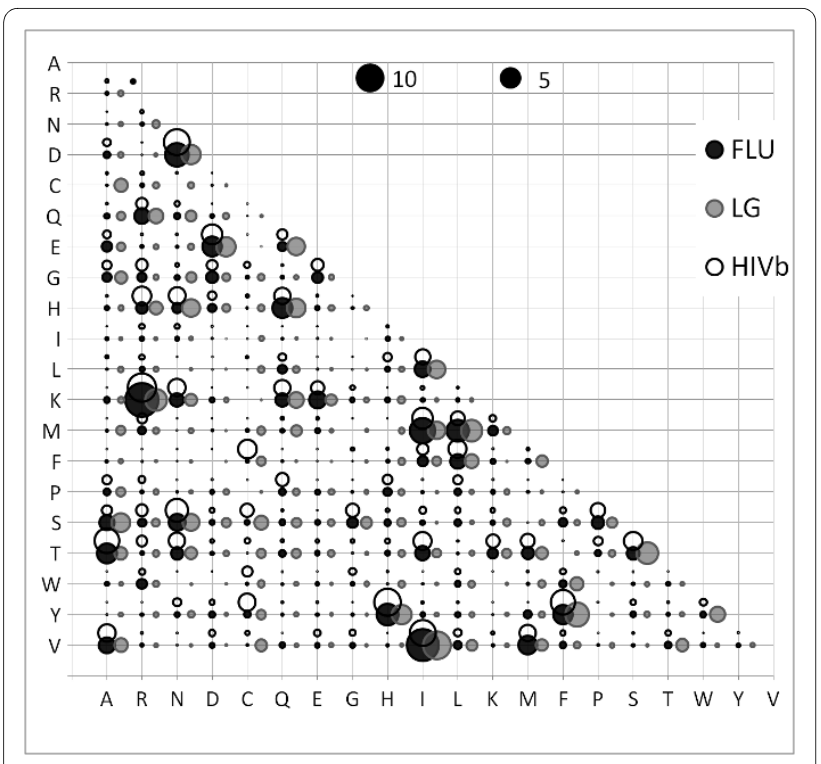

Figure 2 The exchangeability coefficients in FLU, HIVb and LG models. The black bubble at the intersection of line $X$ and column $Y$ presents the exchangeability between amino acid $X$ and amino acid $Y$ in FLU. Similarly, the grey and white bubbles present exchangeabilities between amino acids in the LG and HIVb models, respectively. These bubbles show remarkable differences between these models.

between lysine and cysteine (a neutral, nonpolar amino acid). However, they differ considerably when we look in their relative differences (Figure 3). For example, 41 out of 190 coefficients in FLU are at least 5 times as large as corresponding ones in the HIVb model. Table 2 summarizes the relative differences between FLU and HIVb, LG models.

In a nutshell, FLU is very different from existing models in both amino acid exchangeabilities and frequencies.

\section{FLU performance}

We compared the performance of FLU and other models in constructing maximum likelihood trees for influenza protein alignments. Maximum likelihood trees were constructed by PhyML with 4 discrete gamma rate categories $(+\Gamma=4)$, invariant sites $(+\mathrm{I})$, and $-\mathrm{F} /+\mathrm{F}$ options [18].

\section{Global test}

In the global test, we used FLU and other models to construct maximum likelihood trees for 992 protein alignments of D. Since we estimated and tested FLU on the same data set $\mathbf{D}$, it contains more free parameters than other models, i.e. 208 with $-\mathrm{F}$ option or 189 with $+\mathrm{F}$ option. To compare the performance of FLU and other models, the AIC criterion was used [19].

The average AIC of FLU is higher than that of other models (Table 3). For example, FLU gains 0.3 AIC per site when compared with the second best model, HIVb. In the case where 2 models have the same number of free parameters, 0.3 AIC per site is equivalent to $\sim 45 \log$ likelihood points per alignment of 300 sites. The last column of Table 3 shows the AIC differences between $+\mathrm{F}$ and $-\mathrm{F}$ options. The $+\mathrm{F}$ option would improve the AIC only when the amino acid frequencies of the model are significantly different from the empirical frequencies. However, the $+\mathrm{F}$ option might lead to the loss of AIC due to the penalty of 19 additional free parameters. Table 3 shows that the $+\mathrm{F}$ option did not improve the AIC for most of the models due to the slight difference between the Influenza and the amino acid frequencies of the models, except MtREV, MtMam, and MtArt estimated from mitochondrial proteins. In these cases, the $+\mathrm{F}$ option significantly improved the AIC because of the high difference between the amino acid frequencies of influenza and mitochondrial proteins (correlation $\sim 0.54$ ).

\section{Two-fold cross validation}

In the two-fold cross validation, we randomly divided $\mathbf{D}$ into halves $\mathbf{D}_{1}$ and $\mathbf{D}_{2}$ where either one served as the learning data set and the other acted as the testing data set. Due to the low number of protein types (see Table 4), $\mathbf{D}_{1}$ and $\mathbf{D}_{2}$ might contain alignments of the same protein types. We first estimated $\mathrm{FLU}_{1}\left(\mathrm{FLU}_{2}\right)$ model from $\mathbf{D}_{1}$ $\left(\mathbf{D}_{2}\right)$, and then used $\mathrm{FLU}_{1}\left(\mathrm{FLU}_{2}\right)$ to construct maximum likelihood trees for alignments of $\mathbf{D}_{2}\left(\mathbf{D}_{1}\right)$. Consequently, we obtained 992 maximum likelihood trees inferred using either $\mathrm{FLU}_{1}$ or $\mathrm{FLU}_{2}$. For the sake of simplicity, we denote FLU as the overall model for $\mathrm{FLU}_{1}$ and $\mathrm{FLU}_{2}$ in analyzing the two-fold cross validation. Since learning and testing data sets are independent, there is no penalty for additional free parameters when comparing FLU with other models, i.e., we could directly compare log likelihoods of trees inferred using FLU and other models.

It is clear from Tables 5 and 6 that FLU outperforms all other models. It helps to construct the best likelihood trees for 680 out of 992 alignments (69\%) and the second

Table 2: Relative differences between FLU and HIVb (LG) models.

\begin{tabular}{lcccc} 
& FLU $>$ HIVb & HIVb $>$ FLU & FLU $>$ LG & LG > FLU \\
\hline Twice & 67 & 40 & 20 & 90 \\
Five & 41 & 21 & 2 & 53 \\
\hline
\end{tabular}

The value at the row 'Twice' and column 'FLU>HIVb' indicates the number of exchangeability coefficients in FLU that are at least twice as large as corresponding ones in the HIVb model. Similar explanations can be given for other entries. 


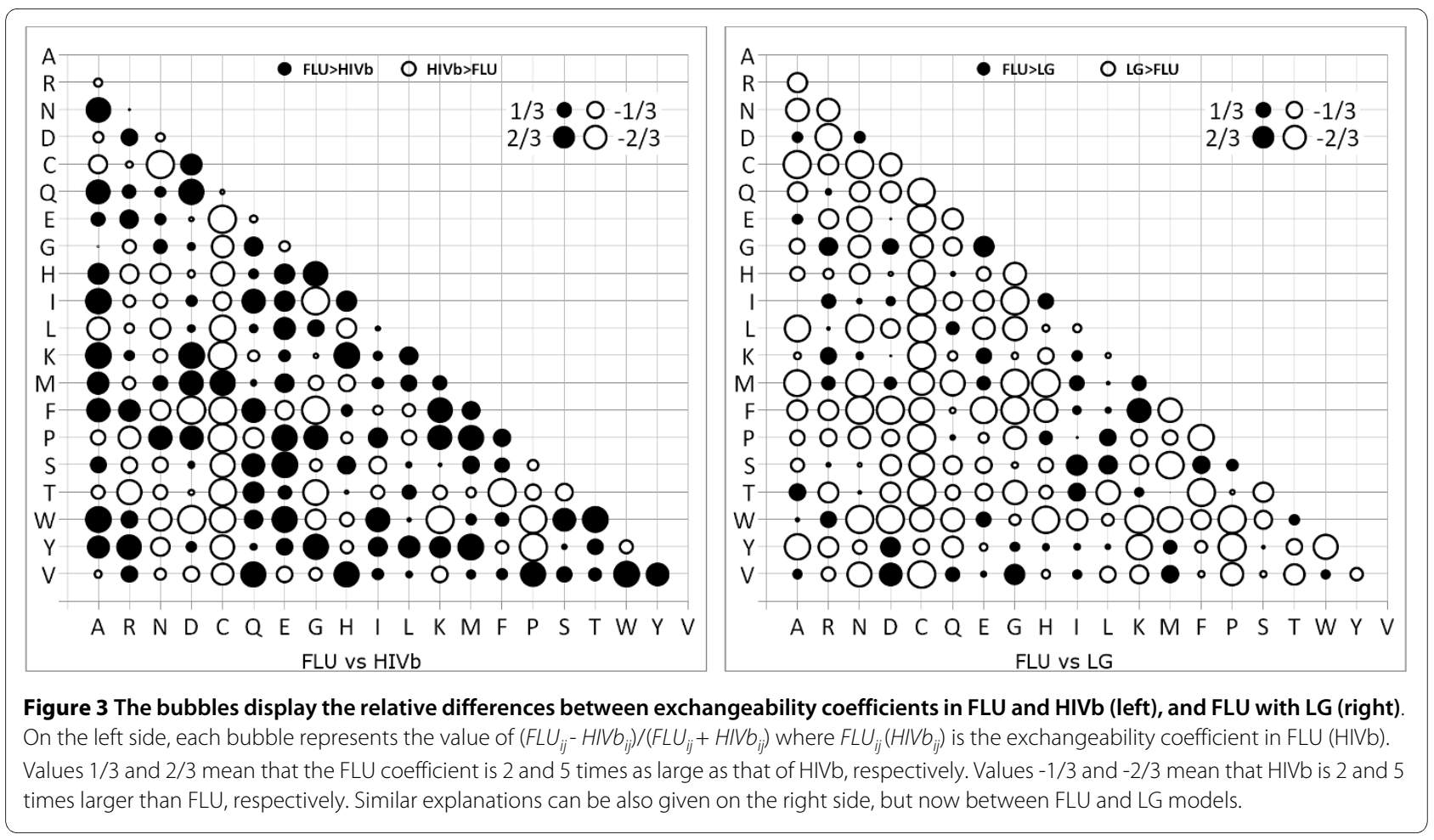

best trees for 131 other alignments (13\%). FLU trees also have the highest average likelihoods, which is $0.14 \log$ likelihood point per site higher than the second best model, HIVb (Table 7). This means that FLU gains about $\sim 42$ log likelihood points on average when applied to an alignment of 300 amino acids. HIV models, as expected, are the second and third best models since they were also estimated from RNA virus proteins. Since HA and NA proteins are the most crucial proteins of influenza viruses, a large number of HA and NA protein sequences

Table 3: Average AIC per site of FLU and other models. FLU has better AIC than other models.

\begin{tabular}{lccc}
\hline & without F option & with F option \\
& $(-\mathbf{F})$ & $\begin{array}{c}\text { difference between } \\
\text { +F and } \mathbf{F} \text { options }\end{array}$ \\
\hline FLU & & -21.09 & -0.08 \\
HIVb & -21.01 & -21.34 & -0.03 \\
JTT & -21.31 & -21.37 & -0.00 \\
HIVw & -21.37 & -21.42 & 0.01 \\
CpREV & -21.43 & -21.54 & -0.05 \\
LG & -21.49 & -21.56 & 0.01 \\
WAG & -21.57 & -21.51 & 0.07 \\
VT & -21.58 & -21.68 & 0.11 \\
Dayhoff & -21.79 & -21.62 & 0.17 \\
DCMut & -21.79 & -21.62 & 0.17 \\
RtREV & -21.79 & -21.70 & 0.10 \\
Blosum62 & -21.80 & -21.82 & 0.03 \\
MtREV & -21.85 & -21.76 & 0.72 \\
MtMam & -22.48 & -21.97 & 0.76 \\
MtArt & -22.73 & -22.15 & 0.71 \\
\hline
\end{tabular}


Table 4: A summary of influenza viruses.

\begin{tabular}{|c|c|c|c|c|}
\hline & Type A & Type B & Type C & proportion (\%) \\
\hline $\mathrm{HA}$ & v & v & $v$ & 30,63 \\
\hline NA & v & v & & 14,67 \\
\hline PA & v & v & & 9,06 \\
\hline PB2 & v & v & v & 8,93 \\
\hline PB1 & v & v & v & 7,97 \\
\hline NS1 & v & v & v & 7,65 \\
\hline NP & v & v & $v$ & 6,87 \\
\hline M2 & v & & & 4,13 \\
\hline NS2 & v & v & v & 3,49 \\
\hline PB1-F2 & v & & & 3,29 \\
\hline M1 & v & v & v & 3,10 \\
\hline NB & & v & & 0,11 \\
\hline BM2 & & v & & 0,04 \\
\hline $\mathrm{CM} 2$ & & & $v$ & 0,03 \\
\hline P3 & & & $\mathrm{v}$ & 0,02 \\
\hline
\end{tabular}

The last column shows proportions of proteins used to estimate the FLU model.

were available to estimate the model (see Table 4). FLU outperforms other models in $\sim 98 \%$ of HA and NA alignments. It is significantly better than HIVb in $\sim 95 \%$ ( $92 \%)$ of HA (NA) alignments. However, it is worse than $\mathrm{HIVb}$ when analyzing M2 and PB1-F2 protein alignments.

The likelihood difference between 2 trees inferred using 2 different models $M_{1}$ and $M_{2}$ might fluctuate due to various error factors, e.g., numerical problems and local optimizations. To assess the statistical significance of the difference between $M_{1}$ and $M_{2}$, we used a simple nonparametric version of the Kishino-Hasegawa $(\mathrm{KH})$ test [20] as used in [6]. As explained in [6], the test avoids any normality assumption and selection bias that would favor one model compared with the other (refer to $[6,21]$ for detailed explanations and calculations). Table 8 shows that FLU is significantly better than other models for the majority of alignments. For example, the $\mathrm{KH}$ test determined $484(\sim 49 \%)$ alignments where FLU trees had significantly higher likelihood values than HIVb trees. The number increases to $731(\sim 74 \%)$ or $907(\sim 92 \%)$ when compared with the JTT and LG, respectively. FLU was significantly worse than one of 14 compared models in only $\sim 7 \%$ of alignments. These comparisons lead to the conclusion that FLU describes the evolution of influenza viruses better than other models, thus resulting in more accurate phylogenetic trees.

\section{Tree analysis}

We observed a large number of alignments where tree topologies of FLU and other models were different (Table
9). For example, FLU trees and HIVb trees are topologically different for 917 ( 92\%) alignments, of which FLU is better than the HIVb for $655(\sim 72 \%)$ alignments.

To measure the difference between 2 tree topologies, we used the Robinson-Fould (RF) distance, which is the number of bi-partitions present in one of the two trees but not the other, divided by the number of possible bipartitions. Thus, the smaller the RF distance between 2 trees, the closer their topologies. Note that the RF ranges from 0.0 to 1.0 .

Figure 4 shows that tree topologies inferred using FLU are highly different from those inferred using other models. For example, the RF distance between FLU trees and HIVb trees is $\sim 0.2(\sim 0.4)$ for about $25 \%(12.5 \%)$ of alignments. The average branch length of FLU trees (0.037) is longer than that of trees inferred using general trees, e.g. LG (0.032), JTT (0.031). This finding indicates that FLU trees capture more hidden substitutions that might have occurred along the branches and therefore might better characterize the evolutionary patterns of influenza viruses than trees inferred using general models (see [22] for discussions on tree length).

\section{Robustness of model}

We investigated the robustness of FLU by measuring the correlations between FLU, FLU 1 and FLU $_{2}$. Table 10 shows extremely high correlations (> 99\%) between FLU, $\mathrm{FLU}_{1}$ and $\mathrm{FLU}_{2}$ in both amino acid frequencies and exchangeability coefficients. Thus, the data set $\mathbf{D}$ is sufficiently large to estimate a robust amino acid substitution model for influenza proteins. 
Table 5: Comparisons of FLU and 14 other models in constructing maximum likelihood trees (-F option).

\begin{tabular}{|c|c|c|c|c|c|c|c|c|c|c|c|c|c|c|c|}
\hline & 1st & 2nd & 3rd & 4th & 5th & 6th & 7th & 8th & 9th & 10th & 11th & 12th & 13th & 14th & 15th \\
\hline FLU & 680 & 129 & 147 & 19 & 2 & 4 & 4 & 4 & 1 & 1 & 1 & 0 & 0 & 0 & 0 \\
\hline $\mathrm{HIVb}$ & 200 & 405 & 198 & 46 & 33 & 64 & 18 & 8 & 7 & 6 & 7 & 0 & 0 & 0 & 0 \\
\hline HIVw & 91 & 115 & 200 & 178 & 64 & 58 & 144 & 20 & 29 & 16 & 16 & 61 & 0 & 0 & 0 \\
\hline JTT & 14 & 274 & 290 & 398 & 14 & 0 & 1 & 0 & 1 & 0 & 0 & 0 & 0 & 0 & 0 \\
\hline LG & 5 & 15 & 26 & 75 & 168 & 394 & 189 & 15 & 64 & 21 & 16 & 4 & 0 & 0 & 0 \\
\hline CpREV & 2 & 25 & 54 & 204 & 542 & 112 & 13 & 20 & 8 & 7 & 4 & 1 & 0 & 0 & 0 \\
\hline WAG & 1 & 28 & 70 & 55 & 134 & 278 & 357 & 43 & 25 & 1 & 0 & 0 & 0 & 0 & 0 \\
\hline Dayhoff & 0 & 1 & 0 & 1 & 8 & 18 & 94 & 196 & 209 & 235 & 200 & 24 & 5 & 1 & 0 \\
\hline VT & 0 & 0 & 3 & 9 & 17 & 30 & 74 & 226 & 192 & 164 & 178 & 71 & 24 & 4 & 0 \\
\hline Blosum62 & 0 & 0 & 3 & 7 & 8 & 18 & 28 & 103 & 84 & 139 & 95 & 436 & 24 & 47 & 0 \\
\hline DCMut & 0 & 0 & 1 & 0 & 1 & 9 & 35 & 103 & 176 & 207 & 249 & 199 & 8 & 4 & 0 \\
\hline RtREV & 0 & 0 & 0 & 0 & 1 & 5 & 29 & 234 & 175 & 174 & 190 & 157 & 14 & 13 & 0 \\
\hline MtMam & 0 & 0 & 0 & 0 & 0 & 2 & 5 & 12 & 10 & 15 & 16 & 14 & 49 & 638 & 230 \\
\hline MtREV & 0 & 0 & 0 & 0 & 0 & 0 & 1 & 8 & 11 & 6 & 20 & 25 & 849 & 69 & 3 \\
\hline MtArt & 0 & 0 & 0 & 0 & 0 & 0 & 0 & 0 & 0 & 0 & 0 & 0 & 19 & 216 & 757 \\
\hline
\end{tabular}

The number on the cell of model $\mathrm{M}$ and column $p$ indicates the number of alignments where $\mathrm{M}$ model stands at the rank $p$ over 15 models tested. For example, FLU model stands at the first rank for 680 out of 992 alignments.

We also examined the influence of the temporal aspect of influenza evolution on FLU. To this end, the data set $\mathbf{D}$ was divided into 2 nearly equal subsets $\mathbf{D}_{t 1}(27,752$ protein sequences before 2004) and $\mathbf{D}_{t 2}(23,397$ protein sequences since 2004). We used subset $\mathbf{D}_{t 1}\left(\mathbf{D}_{t 2}\right)$ to esti- mate model $\mathrm{FLU}_{t 1}\left(\mathrm{FLU}_{t 2}\right)$. FLU $\mathrm{FL}_{1}$ and $\mathrm{FLU}_{t 2}$ were nearly identical (correlation $~ 0.99$ ). Moreover, $\mathrm{FLU}_{t 1}$ and $\mathrm{FLU}_{t 2}$ were highly correlated to FLU (correlation $~ 0.97$ ). The high correlations indicate that the influence of the temporal aspect of influenza evolution on estimating the

Table 6: Comparisons of FLU and 14 other models in constructing maximum likelihood trees (+F option).

\begin{tabular}{|c|c|c|c|c|c|c|c|c|c|c|c|c|c|c|c|}
\hline & $1 \mathrm{st}$ & 2nd & 3rd & 4th & 5th & 6th & 7th & 8th & 9th & 10th & 11th & 12th & 13th & 14th & 15th \\
\hline FLU & 635 & 123 & 202 & 19 & 5 & 2 & 2 & 1 & 2 & 0 & 1 & 0 & 0 & 0 & 0 \\
\hline HIVb & 196 & 375 & 105 & 109 & 61 & 25 & 21 & 22 & 22 & 16 & 35 & 5 & 0 & 0 & 0 \\
\hline HIVw & 148 & 146 & 290 & 73 & 36 & 41 & 22 & 11 & 17 & 56 & 36 & 93 & 19 & 3 & 1 \\
\hline $\mathrm{JTT}$ & 6 & 168 & 218 & 540 & 23 & 20 & 9 & 5 & 1 & 2 & 0 & 0 & 0 & 0 & 0 \\
\hline MtREV & 3 & 1 & 2 & 9 & 77 & 127 & 102 & 66 & 43 & 38 & 115 & 91 & 307 & 10 & 1 \\
\hline MtMam & 2 & 4 & 6 & 7 & 52 & 62 & 53 & 60 & 42 & 62 & 39 & 92 & 71 & 343 & 97 \\
\hline WAG & 1 & 166 & 124 & 52 & 33 & 96 & 146 & 130 & 89 & 63 & 55 & 25 & 12 & 0 & 0 \\
\hline CpREV & 1 & 3 & 5 & 18 & 451 & 159 & 158 & 64 & 95 & 28 & 4 & 3 & 3 & 0 & 0 \\
\hline VT & 0 & 3 & 11 & 21 & 34 & 35 & 46 & 80 & 83 & 135 & 73 & 101 & 206 & 151 & 13 \\
\hline LG & 0 & 1 & 16 & 110 & 131 & 240 & 134 & 83 & 140 & 53 & 42 & 40 & 2 & 0 & 0 \\
\hline Dayhoff & 0 & 1 & 11 & 19 & 60 & 93 & 151 & 227 & 145 & 147 & 91 & 28 & 13 & 5 & 1 \\
\hline Blosum62 & 0 & 1 & 1 & 3 & 1 & 2 & 5 & 11 & 25 & 20 & 115 & 192 & 106 & 203 & 307 \\
\hline DCMut & 0 & 0 & 1 & 11 & 24 & 73 & 108 & 153 & 213 & 143 & 145 & 88 & 26 & 6 & 1 \\
\hline MtArt & 0 & 0 & 0 & 1 & 3 & 1 & 5 & 4 & 4 & 5 & 12 & 32 & 137 & 219 & 569 \\
\hline RtREV & 0 & 0 & 0 & 0 & 1 & 16 & 30 & 75 & 71 & 224 & 229 & 202 & 90 & 52 & 2 \\
\hline
\end{tabular}

The number on the cell of model $\mathrm{M}$ and column $p$ indicates the number of alignments where $\mathrm{M}$ model stands at the rank $p$ over 15 models tested. For example, FLU model stands at the first rank for 635 out of 992 alignments. 
Table 7: Comparisons of FLU and 14 other models in constructing maximum likelihood trees.

\begin{tabular}{lcc}
\hline & $\begin{array}{c}\text { LogLK/site } \\
\text { without F option (-F) }\end{array}$ & $\begin{array}{c}\text { LogLK/site } \\
\text { with F option (+F) }\end{array}$ \\
\hline FLU & & -10.49 \\
HIVb & -10.51 & -10.61 \\
HIVw & -10.65 & -10.65 \\
JTT & -10.71 & -10.63 \\
LG & -10.68 & -10.82 \\
cpREV & -10.78 & -10.93 \\
WAG & -10.74 & -10.70 \\
Dayhoff & -10.78 & -10.71 \\
VT & -10.89 & -10.78 \\
Blosum62 & -10.89 & -10.72 \\
DCMut & -10.92 & -10.75 \\
RtREV & -10.89 & -10.85 \\
MtMam & -10.89 & -10.75 \\
MtREV & -11.36 & -11.01 \\
MtArt & -11.23 & -10.79 \\
\hline FLU & -11.42 & \\
\hline
\end{tabular}

FLU trees have the highest average likelihoods.

amino acid substitution model is insignificant. Thus, FLU is applicable to analyze both old and recent influenza proteins.

\section{Conclusions}

We propose the FLU model that has been specifically estimated for modeling the evolution of influenza viruses. Analyses revealed significant differences between FLU and existing models in both amino acid frequencies and exchangeability coefficients. Experiments showed that
FLU better characterizes the evolutionary patterns of influenza viruses than general models.

Both the global test and 2-fold cross validation confirmed that FLU is better than existing models in constructing maximum likelihood trees. Using the $\mathrm{KH}$ test, FLU proved significantly better than other models for a majority of alignments tested. Nevertheless, there were a few alignments (typically from M2 and PB1-F2 proteins) where FLU was significantly worse than the HIV-specific models or general models, e.g. LG, or JTT. In this study,

Table 8: Pairwise comparisons between FLU and HIVb, HIVw, JTT, LG models.

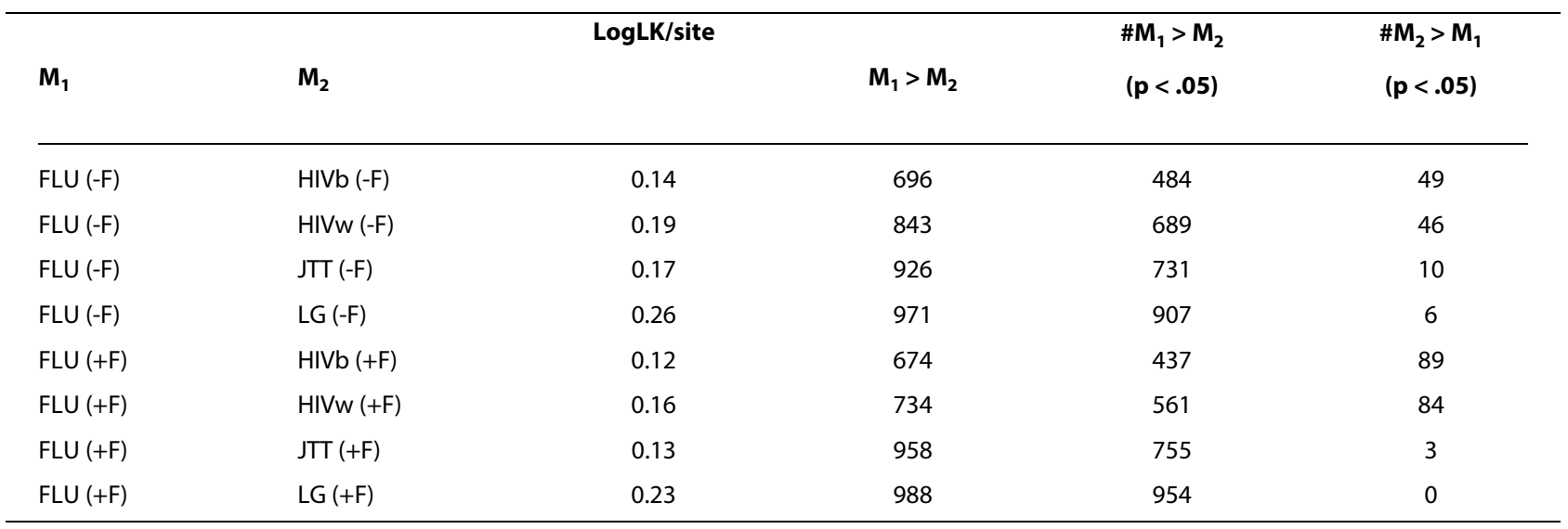

LogLK/site: the log likelihood difference between trees inferred using $M_{1}$ and $M_{2}$; a positive (negative) value means $M_{1}$ is better (worse) than $M_{2}$. $\# M_{1}>M_{2}$ : the number of alignments among 992 alignments where $M_{1}$ results in better likelihood value than $M_{2}$. $\# M_{1}>M_{2}(p<0.05)$ : the number of alignments where the Kishino-Hasegawa test indicates that $M_{1}$ is significantly better than $M_{2} . \# M_{2}>M_{1}(\mathrm{p}<0.05)$ : the same as \#M $>M_{2}$, but now $M_{2}$ is significantly better than $M_{1}$. 
Table 9: Pairwise comparisons between FLU and HIVb, HIVw, JTT, LG models.

\begin{tabular}{|c|c|c|c|c|}
\hline$M_{1}$ & $M_{2}$ & $\# T_{1}>T_{2}$ & $\begin{array}{l}\# T_{1}>T_{2} \\
(p<.05)\end{array}$ & $\begin{array}{l}\# T_{2}>T_{1} \\
(p<.05)\end{array}$ \\
\hline FLU (-F) & HIVb (-F) & $655 / 917$ & 454 & 40 \\
\hline FLU (-F) & HIVw (-F) & $792 / 932$ & 655 & 41 \\
\hline FLU (-F) & $\mathrm{JTT}(-\mathrm{F})$ & $890 / 938$ & 710 & 6 \\
\hline FLU (-F) & LG (-F) & $921 / 935$ & 868 & 5 \\
\hline $\mathrm{FLU}(+\mathrm{F})$ & $\mathrm{HIVb}(+\mathrm{F})$ & $627 / 916$ & 412 & 83 \\
\hline $\mathrm{FLU}(+\mathrm{F})$ & $\mathrm{HIVw}(+\mathrm{F})$ & $701 / 932$ & 540 & 78 \\
\hline $\mathrm{FLU}(+\mathrm{F})$ & $\mathrm{JTT}(+\mathrm{F})$ & $887 / 912$ & 705 & 3 \\
\hline $\mathrm{FLU}(+\mathrm{F})$ & LG (+F) & $922 / 924$ & 897 & 0 \\
\hline
\end{tabular}

amino acid sequences were aligned by Muscle [23] to produce alignments that serve as inputs for estimating FLU. Recently, Liu et al. [24] proposed a method for coestimating sequence alignments and phylogenetic trees, and showed that it improved tree and alignment accuracy compared with 2-phase methods for large DNA data sets. Although previous studies showed that models estimated using near-optimal phylogenetic trees are relatively stable [[10], and references therein], it would be interesting to assess the influence of the coestimation method on the estimation of amino acid substitution models in future work. The occurrence of homologous recombination within influenza virus genes has been reported, however, it is rare and controversial $[25,26]$. Therefore, the FLU was estimated in a standard phylogenetic framework. The effect of the homologous recombination, if it occurs at all, on the FLU model would be discovered in future work. In summary, FLU model is useful for any influenza protein analysis system that demands an accurate

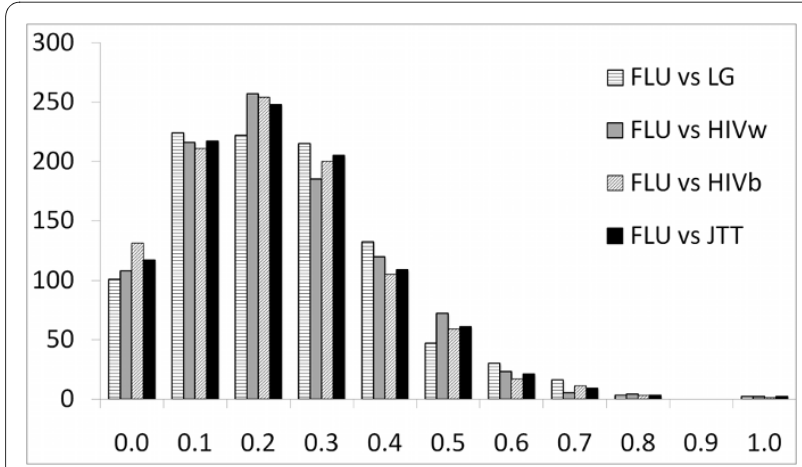

Figure 4 The Robinson-Foulds distance between trees inferred using FLU and HIVb (LG, JTT, HIVw) models. The horizontal axis indicates the RF distance between 2 tree topologies, whereas the vertical axis indicates the number of alignments. description of amino acid substitutions. It should enhance our understanding of the evolution, transmission and infection processes of influenza viruses.

\section{Methods}

Data

Influenza viruses are RNA viruses from the Orthomyxoviridae family, which is divided into 3 types: influenzas $A$, $B$, and $C$. Influenza A viruses frequently cause serious epidemics and pandemics, such as Spanish flu H1N1, Asian flu H2N2, Hong Kong flu H3N2, or avian flu H5N1 (see Table 4 for a short summary of influenza viruses). Influenza viruses have been isolated since the beginning of the 20th century, and a huge number of their proteins have been sequenced and stored at the NCBI $[13,16]$.

To estimate the amino acid substitution model for influenza viruses, we downloaded the entire influenza database at NCBI (July 26th 2009 version) [16], including 112,450 protein sequences $(103,626$ for A; 7,892 for B; and 932 for $C$ ). The sequences were processed before estimating the model.

- Cleaning step: Only distinct sequences were kept. The set consisted of 51,061 sequences, i.e. 46,909 for A; 3,845 for B; and 307 for C.

- Dividing step: These distinct sequences were randomly divided into small groups such that each group contained from 5 to 100 homologous sequences (the same protein type) of the same virus type. This resulted in 1046 groups.

- Aligning step: The 1046 groups were aligned by Muscle, a multiple alignment program [23]. The alignments were cleaned by the GBLOCKS [27] to eliminate sites containing many gaps. We selected 992 alignments which contain at least 5 sequences and 50 sites for estimating the model. 


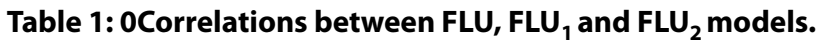

\begin{tabular}{|c|c|c|}
\hline & exchangeability matrix & frequency vector \\
\hline FLU vs FLU & $99.95 \%$ & $99.98 \%$ \\
\hline $\mathrm{FLU}$ vs $\mathrm{FLU}_{2}$ & $99.95 \%$ & $99.98 \%$ \\
\hline $\mathrm{FLU}_{1} \mathrm{vs} \mathrm{FLU}_{2}$ & $99.81 \%$ & $99.94 \%$ \\
\hline
\end{tabular}

The exchangeability (frequency) column gives the correlations between exchangeability matrices (frequency vectors) of these models.

\section{Model}

We assume, as usual, that amino acid sites evolve independently, and the process has remained constant throughout the course of evolution. The substitution process between amino acids is modeled by a time-homogeneous, time-continuous, time-reversible, and stationary Markov process [[1,2,28], and references therein]. The central component of the process is the so-called instantaneous substitution rate $20 \times 20$-matrix $\mathbf{Q}=\left\{q_{x y}\right\}$ where $q_{x y}(x \neq y)$ is the number of substitutions from amino acid $x$ to amino acid $y$ per time unit. The diagonal elements $q_{x x}$ are assigned such that the sum of each row equals zero. The matrix $\mathbf{Q}$ can be decomposed into symmetric exchangeability rate matrix $R=\left\{r_{x y}\right\}$ and amino acid frequency vector $\pi=\left\{\pi_{x}\right\}$ such that $q_{x y}=r_{x y} \pi_{y}$ and $q_{x x}=-\Sigma_{y \neq x}$ $q_{x y}$

The likelihood of a multiple sequence alignment $D=$ $\left\{d_{1}, \ldots, d_{n}\right\}$ of $n$ sites given their phylogenetic tree $T$ and the model $\mathbf{Q}$ is

$$
L(T, \mathbf{Q} \mid D)=\prod_{i=1}^{n} L\left(T, \mathbf{Q} \mid d_{i}\right)
$$

where $L\left(T, \mathbf{Q} \mid d_{i}\right)$ is the likelihood of site $d_{i}$ given tree $T$ and model $\mathbf{Q}$ that can be efficiently calculated by a pruning algorithm [29].

In Equation 1, we assumed the same substitution rate across amino acid sites. To incorporate the variability of substitution rates across sites we used the combination of invariant model [30,31] and $\Gamma$-distribution model [32]. The heterogeneous rate model $\mathbf{r}$ assumes a fraction $\theta_{\text {inv }}$ of sequence sites to be invariant, and other sites are variant with global substitution rates following the $\Gamma$-distribution [33].

The likelihood of $D$ given the phylogenetic tree $T$, substitution model $\mathbf{Q}$, and rate model $\mathbf{r}$ is computed as

$$
\begin{aligned}
& L(T, \mathbf{Q}, \mathbf{r} \mid D)=\prod_{i=1}^{n} L\left(T, \mathbf{Q}, \mathbf{r} \mid d_{i}\right) \\
& =\prod_{i=1}^{n}\left[\theta_{\text {inv }} L\left(i n v \mid d_{i}\right)+\left(1-\theta_{\text {inv }}\right) \sum_{c=1}^{C} \frac{1}{C} L\left(r_{c} T, \mathbf{Q} \mid d_{i}\right)\right]
\end{aligned}
$$

where $L\left(i n v \mid d_{i}\right)$ is the likelihood of site $d_{i}$ following the invariant model, that is, $L\left(i n v \mid d_{i}\right)$ is equal to $\pi_{x}$ if site $d_{i}$ is constant and contains only amino acid $x$, otherwise zero when the site $d_{i}$ is not constant; $r_{c} T$ denotes the tree $T$ with all branch lengths being multiplied by $r_{c}$.

\section{Model estimation}

Given a set of $m$ protein alignments $\mathbf{D}=\left\{D_{1}, \ldots, D_{m}\right\}$, the substitution model $\mathbf{Q}$ can be estimated by the counting or the maximum likelihood approach [[1], and references therein]. A number of studies have shown that the maximum likelihood approach can avoid systematic errors and makes more efficient use of information in the protein alignments compared with the counting approach [10]. We applied the maximum likelihood approach, introduced by Le and Gascuel in [6], to estimate the model $\mathbf{Q}$.

The model $\mathbf{Q}$ is estimated by maximizing the likelihood $L(\mathbf{D})$ :

$$
\mathbf{Q}=\underset{\mathbf{Q}^{\prime}}{\arg \max }\left\{L(\mathbf{D})=\prod_{i=1}^{m} L\left(T_{i}, \mathbf{Q}^{\prime}, \mathbf{r}_{\mathbf{i}} \mid D_{i}\right)\right\}
$$

where $T_{i}$ and $\mathbf{r}_{i}$ are the phylogenetic tree and rate model of the alignment $D_{i}$, respectively. Optimizing the likelihood $L(\mathbf{D})$ is a difficult problem because we have to construct all phylogenetic trees (topologies and branch lengths), $\mathbf{Q}$ coefficients and rate parameters. Fortunately, previous studies discovered that the estimated coefficients of $\mathbf{Q}$ remained nearly unchanged when near-optimal phylogenetic trees and rate parameters were used [[10], and references therein]. Thus, the Equation 2 can be simplified and approximated to:

$$
L(\mathbf{D})=\prod_{i=1}^{m} L\left(\mathbf{Q} \mid \tilde{T}_{i}, \tilde{\mathbf{r}}_{i}, D_{i}\right)
$$

where $\tilde{T}_{i}$ and $\tilde{\mathbf{r}}_{i}$ are near-optimal phylogenetic tree and rate model of $D_{i}$, respectively. We designed a 5-step procedure to estimate the model $\mathbf{Q}$ (see Figure 5): 


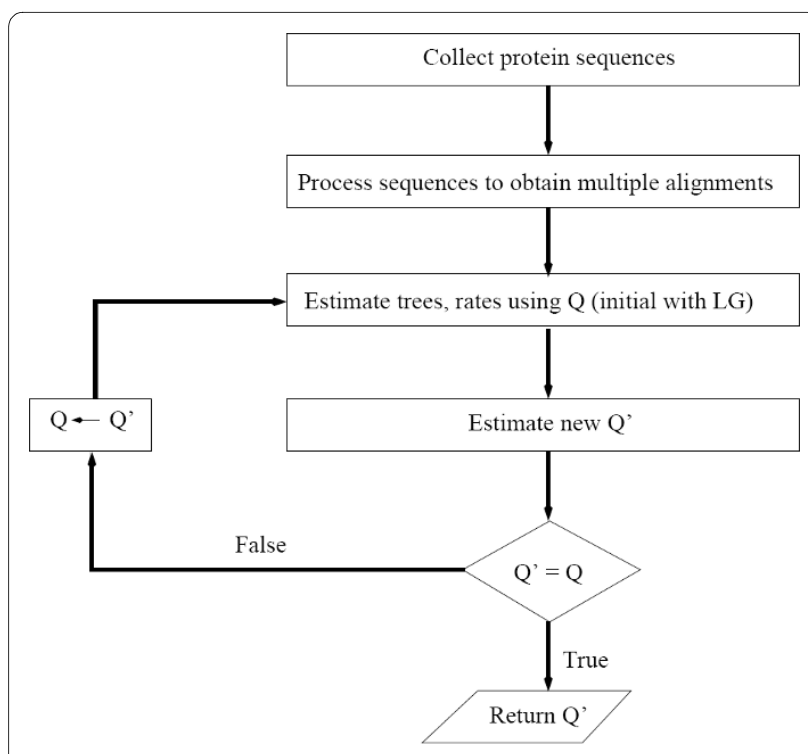

Figure 5 Flowchart to estimate the influenza-specific amino acid substitution model.

- Step 1: Collect all influenza protein sequences from the influenza database at NCBI $(112,450$ protein sequences).

- Step 2: Process retrieved sequences as described in the 'Data' section to obtain 992 multiple alignments.

- Step $3(\mathbf{Q}=L G$ as the default $)$ : Estimate trees, rates, etc., using $\mathbf{Q}$ and the phylogenetic software PhyML [18].

- Step 4: Estimate a new model Q' using the approach introduced in [6] and the XRate software [34].

- Step 5: Compare 2 models $\mathbf{Q}$ and $\mathbf{Q}^{\prime}$. If $\mathbf{Q}^{\prime}$ is nearly identical to $\mathbf{Q}$, return $\mathbf{Q}^{\prime}$ and consider it as the model for influenza viruses. Otherwise, $\mathbf{Q} \quad \mathbf{Q}^{\prime}$ and goto Step 3.

FLU was obtained after two iterations.

\section{Authors' contributions}

CCD, QSL, VSL, and OG discussed ideas. CCD implemented programs, conducted experiments, and wrote the draft manuscript. QSL and VSL designed experiments and revised the manuscript. All authors read and approved the final manuscript.

\section{Acknowledgements}

We would like to express our special thanks to Leopold Parts, and Hang Phan for carefully reading the manuscript. We thank two anonymous reviewers for helpful suggestions. Financial support from Vietnam National Foundation for Science and Technology Development is greatly appreciated.

\section{Author Details}

${ }^{1}$ College of Technology, Vietnam National University Hanoi, 144 Xuan Thuy, Cau Giay, Hanoi, Vietnam, 2Wellcome Trust Sanger Institute, Wellcome Trust Genome Campus, Hinxton, Cambridge, CB10 1SA, UK and ${ }^{3}$ Methodes et Algorithmes pour la Bioinformatique, LIRMM, CNRS, Universite Montpellier II, Montpellier, France

Received: 21 September 2009 Accepted: 12 April 2010

Published: 12 April 2010

\section{References}

1. Felsenstein J: Infering Phylogenies Sunderland, Massachusetts, US: Sinauer Associates; 2004.

2. Ziheng Y: Computational Molecular Evolution 1st edition. Oxford, UK: Oxford University Press; 2006.

3. Opperdoes FR: Phylogenetic analysis using protein sequences. In The Phylogenetics Handbook A Practical Approach to DNA and Protein Phylogeny Edited by: Salemi M, Vandamme AM. Cambridge: Cambridge University Press; 2003:207-235.

4. Setubal C, Meidanis J: Introduction to Computational Molecular Biology 1st edition. Boston, Massachusetts, US: PWS Publishing; 1997.

5. Thorne J: Models of protein sequence evolution and their applications. Currrent Opinion in Genetics and Development 2000, 10:602-605.

6. Le S, Gascuel O: An improved general amino acid replacement matrix. Mol Biol Evol 2008, 25:1307-1320.

7. Dayhoff MO, Schwartz RM, Orcutt BC: A Model of Evolutionary Change in Proteins. In Atlas of Protein Sequence Structure Volume 5. Edited by: Dayhoff MO. Washington DC: National Biomedical Research Foundation; 1978:345-352.

8. Jones DT, Taylor WR, Thornton JM: The rapid generation of mutation data matrices from protein sequences. Comput Appl Biosci 1992, 8:275-282.

9. Adachi J, Hasegawa M: Model of Amino Acid Substitution in Proteins Encoded by Mitochondrial DNA. J Mol Evol 1996, 42:459-468.

10. Whelan S, Goldman N: A General Empirical Model of Protein Evolution Derived from Multiple Protein Families Using a Maximum Likelihood Approach. Mol Biol Evol 2001, 18:691-699.

11. Dimmic MW, Rest JS, Mindell DP, Goldstein RA: rtREV: an amino acid substitution matrix for inference of retrovirus and reverse transcriptase phylogeny. J Mol Evol 2002, 55:65-73.

12. Nickle DC, Heath $L$, Jensen MA, Gilbert PB, Mullins JI, Pond SK: HIV-Specific Probabilistic Models of Protein Evolution. PLOS ONE 2007, 2:e503.

13. Fauci A: Race against time. Nature 2009, 435:423-424.

14. Ghedin E, Sengamalay N, Shumway M, Zaborsky J, Feldblyum T, Subbu V, Spiro D, Sitz J, Koo H, Bolotov P, Dernovoy D, Tatusova T, Bao Y, St George K, Taylor J, Lipman D, Fraser C, Taubenberger J, Salzberg S: Large-scale sequencing of human influenza reveals the dynamic nature of viral genome evolution. Nature 2005, 437:1162-1166.

15. Janies DA, Hill A, Guralnick R, Habib F, Waltari E, Wheeler WC: Genomic Analysis and Geographic Visualization of the Spread of Avian Influenza (H5N1). Systematic Biology 2007, 56:321-329.

16. Bao Y, Bolotov P, Dernovoy D, Kiryutin B, Zaslavsky L, Tatusova T, Ostell J, Lipman D: The Influenza Virus Resource at the National Center for Biotechnology Information. J Virol 2008, 82:596-601.

17. Nguyen T, Nguyen T, Vijaykrishna D, Webster R, Guan Y, Malik Peiris J, Smith G: Multiple Sublineages of Influenza A Virus (H5N1), Vietnam, 2005-2007. Emerging Infectious Diseases 2008, 14:632-636.

18. Guindon S, Gascuel O: A Simple, Fast and Accurate Algorithm to Estimate Large Phylogenies by Maximum Likelihood. Syst Biol 2003, 52:696-704

19. Akaike $\mathrm{H}$ : A new look at the statistical model identification. IEEE Trans Automat Contr 1974, 19:716-722.

20. Kishino $\mathrm{H}$, Hasegawa M: Evaluation of the maximum likelihood estimate of the evolutionary tree topologies from DNA sequence data, and the branching order in hominoidea. J Mol Evol 1989, 29:170-179.

21. Goldman N, Anderson J, Rodrigo A: Likelihood-based tests of topologies in phylogenetics. Syst Biol 2000, 49:652-670.

22. Pagel M, Meade A: Mixture models in phylogenetic inference. In Mathematics of evolution and phylogeny Edited by: Gascuel O. Oxford, UK: Oxford University Press; 2005:121-142.

23. Edgar RC: MUSCLE: multiple sequence alignment with high accuracy and high throughput. Nucl Acids Res 2004, 32:1792-1797.

24. Kevin L, Sindhu R, Serita N, Randal L, Tandy W: Rapid and Accurate LargeScale Coestimation of Sequence Alignments and Phylogenetic Trees. Science 2009, 324:1561-1564.

25. Boni M, Zhou Y, Taubenberger J, Holmes E: Homologous Recombination is Very Rare or Absent in Human Influenza A Virus. Journal Virology 2008, 82:4807-4811

26. He CQ, Xie ZX, Han GZ, Dong JB, Wang D, Liu JB, Ma LY, Tang XF, Liu XP, Pang YS, Li GR: Homologous Recombination as an Evolutionary Force in the Avian Influenza A Virus. Mol Bio Evol 2009, 26:177-187. 
27. Castresana J: Selection of conserved blocks from multiple alignments for their use in phylogenetic analysis. Molecular Biology and Evolution 2000, 17:540-552.

28. Strimmer K, Haeseler AV: Nucleotide Substitution Models. In The Phylogenetics Handbook A Practical Approach to DNA and Protein Phylogeny Edited by: Salemi M, Vandamme AM. Cambridge: Cambridge University Press; 2003:72-100.

29. Felsenstein J: Evolutionary trees from DNA sequences: A maximum likelihood approach. Journal of Molecular Evolution 1981, 17:368-376.

30. Fitch WM, Margoliash E: A method for estimating the number of invariant amino acid position in a gene using cytochrome $\mathrm{c}$ as a model case. Biochem Gene 1967, 1:65-71.

31. Churchill GA, Haeseler AV, Navidi WC: Sample Size for Phylogenetic Inference. Mol Biol Evol 1992, 9:753-769.

32. Yang Z: Maximum-likelihood estimation of phylogeny from DNA sequences when substitution rates differ over sites. Mol Biol Evol 1993 10:1396-1401.

33. Gu X, Fu YX, Li WH: Maximum Likelihood Estimation of the Heterogeneity of Substitution Rate among Nucleotide Sites. Mol Biol Evol 1995, 12:546-557.

34. Klosterman P, Uzilov A, Bendana Y, Bradley R, Chao S, Kosiol C, Goldman N, Holmes I: XRate: a fast prototyping, training and annotation tool for phylo-grammars. BMC Bioinformatics 2006, 7:428.

doi: 10.1186/1471-2148-10-99

Cite this article as: Dang et al., FLU, an amino acid substitution model for influenza proteins BMC Evolutionary Biology 2010, 10:99

Submit your next manuscript to BioMed Central and take full advantage of:

- Convenient online submission

- Thorough peer review

- No space constraints or color figure charges

- Immediate publication on acceptance

- Inclusion in PubMed, CAS, Scopus and Google Scholar

- Research which is freely available for redistribution

Submit your manuscript at www.biomedcentral.com/submit
C) Biomed Central 